\title{
A NOTE ON THE PAPER "OPTIMALITY CONDITIONS FOR NONSMOOTH INTERVAL-VALUED AND MULTIOBJECTIVE SEMI-INFINITE PROGRAMMING"
}

\author{
Nazih Abderrazzak Gadhi and Aissam Ichatouhane*
}

\begin{abstract}
A nonsmooth semi-infinite interval-valued vector programming problem is solved in the paper by Jennane et al. (RAIRO:OR 55 (2021) 1-11.). The necessary optimality condition obtained by the authors, as well as its proof, is false. Some counterexamples are given to call into question some results on which the main result (Jennane et al. [6] Thm. 4.5) is based. For the convenience of the reader, we correct the faulty in those results, propose a correct formulation of Theorem 4.5 , and give also a short proof.
\end{abstract}

Mathematics Subject Classification. 58E35, 90C46, 90C29, $49 \mathrm{~J} 52$.

Received July 25, 2020. Accepted September 22, 2020.

\section{INTRODUCTION}

Optimization problems are significant since they are applied to many research fields. If the coefficients of objective and constraint functions are taken as closed intervals, we obtain interval-valued optimization problems. These problems may provide the possibility for a different choice that will address uncertainty in optimization. Optimality conditions and duality results for interval-valued nonlinear programming problems are studied by many researchers $[1,2,5,6,8,10]$. In the paper [6], Jennane et al. investigated the following semi-infinite intervalvalued vector program

$$
(2):\left\{\begin{array}{l}
\operatorname{Min}\left\{F(x)=\left(F_{1}(x), \ldots, F_{p}(x)\right): x \in \Omega\right\} \\
\Omega=\left\{x \in \mathbb{R}^{n}: G_{t}(x) \leq_{L U} A_{t}, \forall t \in T\right\}
\end{array}\right.
$$

where $T$ is an arbitrary (possibly infinite) index set, $A_{t}=\left[A_{t}^{L}, A_{t}^{U}\right] \subseteq \mathbb{R}$ is a closed interval for all $t \in T$, $F_{k}=\left[F_{k}^{L}, F_{k}^{U}\right]$ and $G_{t}=\left[G_{t}^{L}, G_{t}^{U}\right]$ are interval-valued functions defined on $\mathbb{R}^{n}$ for all $k \in I:=\{1, \ldots, p\}$ and $t \in T$.

Under a nonsmooth constraint qualification $(A C Q)$ given in terms of convexifactors, the authors established necessary optimality condition for Problem (2). The main result, Jennane et al. [6] Theorem 4.5, is based on Lemmas 3.3 and 3.4 of [6].

In this note, we show that Jennane et al. [6] Lemmas 3.3 and 3.4 need correction. In support of our remarks, some counterexamples are given (see Examples 3.1, 3.2 and 3.6) and some reasoning's mistakes are highlighted

Keywords. Convexifactor, constraint qualifications, interval-valued functions, optimality conditions.

LAMA, FSDM, Department of Mathematics, Sidi Mohamed Ben Abdellah University, Fes, Morocco.

${ }^{*}$ Corresponding author: ichatouhane22@gmail.com 
(see Rems. 3.3 to 3.5 and 3.7). Since those intermediate lemmas are integral parts of the proof of the main result, necessary optimality conditions obtained by the authors are, in our view, not valid. Theorem 4.6 is actually a corrected version of Theorem 4.5 of [6].

The rest of the paper is organized in this way: Section 2 contains basic definitions and preliminary material. Counterexamples and comments are given in Section 3. Section 4 addresses our main results (corrected optimality conditions). A conclusion is given in Section 5 .

\section{Preliminaries}

Throughout this paper, $\mathbb{R}^{n}$ is the usual $n$-dimensional Euclidean space with a norm $\|\cdot\|$. We denote by $\langle\cdot, \cdot\rangle$ and $\mathbb{R}_{+}^{n}$ the inner product and the non-negative orthant of $\mathbb{R}^{n}$ defined by

$$
\mathbb{R}_{+}^{n}=\left\{\left(x_{1}, \ldots, x_{n}\right) \in \mathbb{R}^{n}: x_{i} \geq 0\right\} .
$$

For a subset $S$ of $\mathbb{R}^{n}$, the sets cl $S$ and co $S$ stand for the closure of $S$ and the convex hull of $S$, respectively.

Let $S$ be a subset of $\mathbb{R}^{n}$ and $x \in \operatorname{cl} S$. The negative polar cone of $S$ is defined by

$$
S^{\circ}:=\left\{v \in \mathbb{R}^{n}:\langle s, v\rangle \leq 0, \forall s \in S\right\} .
$$

The tangent and normal cones to $S$ at $x$ are given by

$$
T_{S}(x)=\left\{v \in \mathbb{R}^{n}: \exists t_{n} \downarrow 0 \text { and } \exists v_{n} \rightarrow v \text { such that } x+t_{n} v_{n} \in S, \forall n \in \mathbb{N}\right\}
$$

and

$$
N_{S}(x)=\left\{\xi \in \mathbb{R}^{n}:\langle\xi, v\rangle \leq 0, \forall v \in T_{S}(x)\right\}=T_{S}(x)^{\circ} .
$$

Let $\mathcal{I}$ be the set of all closed and bounded intervals in $\mathbb{R}$ and let $A=\left[a^{L}, a^{U}\right] \in \mathcal{I}$ and $B=\left[b^{L}, b^{U}\right] \in \mathcal{I}$. In [6], Jennane et al. considered the partial ordering for intervals defined by:

- $A \leq_{L U} B$ iff $a^{L} \leq b^{L}$ and $a^{U} \leq b^{U}$, with at least one strict inequality.

$-A<_{L U} B$ iff $a^{L}<b^{L}$ and $a^{U}<b^{U}$.

Notice that $A=\left(A_{1}, \ldots, A_{p}\right)$ is called an interval-valued vector if $A_{k}=\left[a_{k}^{L}, a_{k}^{U}\right] \in \mathcal{I}$ for each $k \in I$. For two interval-valued vectors $A=\left(A_{1}, \ldots, A_{p}\right)$ and $B=\left(B_{1}, \ldots, B_{p}\right)$, we have:

- $A \leq_{L U} B$ iff $A_{k} \leq_{L U} B_{k}$ for each $k \in I$ except at least one index for which the inequality is strict.

- $A<_{L U} B$ iff $A_{k}<_{L U} B_{k}$ for each $k \in I$.

A feasible solution $\bar{x} \in \Omega$ is an efficient solution to Problem (2) if there exist no $x \in \Omega$ such that

$$
F(x) \leq_{L U} F(\bar{x}) .
$$

A feasible solution $\bar{x} \in \Omega$ is a weak efficient solution to Problem (2) if there exist no $x \in \Omega$ such that

$$
F(x)<_{L U} F(\bar{x}) .
$$

Definition 2.1 ([4]). A nonempty set $S \subseteq \mathbb{R}^{n}$ is said to be locally star-shaped at $\bar{x} \in S$, if corresponding to $\bar{x}$ and each $x \in S$, there exists some scalar $a(\bar{x}, x) \in(0,1]$ such that

$$
\bar{x}+\lambda(x-\bar{x}) \in S, \text { for all } \lambda \in(0, a(\bar{x}, x)) .
$$

If $a(\bar{x}, x)=1$ for each $x \in S$, then $S$ is said to be star-shaped at $\bar{x}$.

As examples of locally star-shaped sets, open sets and convex sets are locally star-shaped at each of their elements, and cones are locally star-shaped at the origin. If $S$ is closed and is locally star-shaped at each $\bar{x} \in S$, then $S$ is convex [9]. 
Remark 2.2 ([8]). There exist locally star-shaped sets (at some $\bar{x}$ ) that are not star-shaped $($ at $\bar{x})$. For example,

$$
S=\mathbb{R}^{2} \backslash\left\{(x, y) \in \mathbb{R}^{2}: y=x^{2} \text { and } x \neq 0\right\}
$$

is locally star-shaped at $\bar{x}=(0,0)$ and is not star-shaped at $\bar{x}$.

Now, we recall the definitions related to convexifactors given by Jeyakumar and Luc [7] and Dutta and Chandra [3]. Let $f: \mathbb{R}^{n} \rightarrow \mathbb{R} \cup\{+\infty\}$ be a given function and let $x \in \mathbb{R}^{n}$ where $f(x)$ is finite. The expressions

$$
f_{d}^{-}(x, v)=\liminf _{t \searrow 0}[f(x+t v)-f(x)] / t
$$

and

$$
f_{d}^{+}(x, v)=\limsup _{t \searrow 0}[f(x+t v)-f(x)] / t
$$

signify, respectively, the lower and upper Dini directional derivatives of $f$ at $x$ in the direction $v$.

Definition 2.3 ([3]). The function $f: \mathbb{R}^{n} \rightarrow \mathbb{R} \cup\{+\infty\}$ is said to have an upper convexifactor $\partial^{u} f(x)$ at $x$ if $\partial^{u} f(x) \subseteq \mathbb{R}^{n}$ is closed and, for each $v \in \mathbb{R}^{n}$,

$$
f_{d}^{-}(x, v) \leq \sup _{x^{*} \in \partial^{u} f(x)}\left\langle x^{*}, v\right\rangle .
$$

The function $f: \mathbb{R}^{n} \rightarrow \mathbb{R} \cup\{+\infty\}$ is said to have an upper semiregular convexifactor (USRC) $\partial^{\text {us }} f(x)$ at $x$ if $\partial^{\text {us }} f(x)$ is an upper convexifactor at $x$ and, for each $v \in \mathbb{R}^{n}$,

$$
f_{d}^{+}(x, v) \leq \sup _{x^{*} \in \partial^{\text {us }} f(x)}\left\langle x^{*}, v\right\rangle .
$$

Remark 2.4 ([3]). The Clarke, Michel-Penot and Mordokhovich subdifferentials are upper semiregular convexifactors of $f$ when $f$ is a locally Lipschitz function. However, the convex hull of an upper semiregular convexifactor of a locally Lipschitz function may be strictly contained in both the Clarke and the Michel-Penot subdifferentials.

\section{Counterexamples And COMments}

The following examples show that Lemma 3.3 of [6] is not correct.

Example 3.1. Consider the optimization Problem (2) where

$$
T:=\mathbb{N}, G_{t}^{L}(x):=-x^{2}-\frac{1}{t}, G_{t}^{U}(x):=x^{2}+\frac{1}{t}, A_{t}^{L}:=-\frac{1}{t} \text { and } A_{t}^{U}:=\frac{1}{t}, \forall t \in T .
$$

- On the one hand, $\Omega=\emptyset$. Indeed, since

$$
x \in \Omega \Longleftrightarrow\left(G_{t}^{L}(x) \leq A_{t}^{L} \text { and } G_{t}^{U}(x)<A_{t}^{U}\right) \text { or }\left(G_{t}^{L}(x)<A_{t}^{L} \text { and } G_{t}^{U}(x) \leq A_{t}^{U}\right), \text { for all } t \in T
$$

we get

$$
x \in \Omega \Longleftrightarrow\left(-x^{2}-\frac{1}{t} \leq-\frac{1}{t} \text { and } x^{2}+\frac{1}{t}<\frac{1}{t}\right) \text { or }\left(-x^{2}-\frac{1}{t}<-\frac{1}{t} \text { and } x^{2}+\frac{1}{t} \leq \frac{1}{t}\right) \text {, for all } t \in T \text {. }
$$

Then,

Consequently,

$$
x \in \Omega \Longleftrightarrow\left(-x^{2} \leq 0 \text { and } x^{2}<0\right) \text { or }\left(-x^{2}<0 \text { and } x^{2} \leq 0\right) .
$$

Finally, $\Omega=\emptyset$.

$$
x \in \Omega \Longleftrightarrow\left(-x^{2}<0 \text { and } x^{2} \leq 0\right) \Longleftrightarrow(x \neq 0 \text { and } x=0) .
$$


- On the other hand, since

$$
g_{t}(x):=\max \left(G_{t}^{L}(x)-A_{t}^{L}, G_{t}^{U}(x)-A_{t}^{U}\right), \forall t \in T,
$$

we have

$$
g_{t}(x)=\max \left(-x^{2}, x^{2}\right)=x^{2}, \forall t \in T
$$

Consequently,

$$
\left\{x \in \mathbb{R}: g_{t}(x) \leq 0, \forall t \in T\right\}=\{0\} .
$$

Clearly, the feasible set of Problem (2) is not equal to $\left\{x \in \mathbb{R}: g_{t}(x) \leq 0, \forall t \in T\right\}$.

Even the example provided by the authors in Section 6 shows that Lemma 3.3 of [6] is not correct.

Example 3.2. Consider the optimization Problem (2) where

$$
T:=[-1,1], G_{t}^{L}(x):=t x_{1}-x_{2}, G_{t}^{U}(x):=t x_{1}-x_{2}+1, A_{t}^{L}(x):=0 \text { and } A_{t}^{U}(x):=1, \forall t \in T .
$$

- On the one hand, since

$$
x \in \Omega \Longleftrightarrow t x_{1}-x_{2} \leq 0 \text { and } t x_{1}-x_{2}<0, \forall t \in T,
$$

we obtain

$$
\Omega=\left\{\left(x_{1}, x_{2}\right) \in \mathbb{R}^{2}: x_{2}>\left|x_{1}\right|\right\}
$$

- On the other hand, since

$$
g_{t}(x)=t x_{1}-x_{2}, \forall t \in T
$$

we have

$$
\left\{x \in \mathbb{R}: g_{t}(x) \leq 0, \forall t \in T\right\}=\left\{\left(x_{1}, x_{2}\right) \in \mathbb{R}^{2}: x_{2} \geq\left|x_{1}\right|\right\} .
$$

Clearly, the feasible set of Problem (2) is not equal to $\left\{x \in \mathbb{R}: g_{t}(x) \leq 0, \forall t \in T\right\}$. Notice that $(0,0) \notin \Omega$ while $(0,0) \in\left\{x \in \mathbb{R}: g_{t}(x) \leq 0, \forall t \in T\right\}$.

Remark 3.3. The feasible set of Problem (2) may be strictly included in $\left\{x \in \mathbb{R}: g_{t}(x) \leq 0, \forall t \in T\right\}$. Consequently, the set of weak efficient solutions of (2) is not necessarily equal to the set of weak minima of

$$
\text { (4) : }\left\{\begin{array}{c}
\operatorname{Min}\left\{f(x)=\left(f_{1}(x), f_{2}(x)\right): x \in \widetilde{\Omega}\right\}, \\
\widetilde{\Omega}=\left\{x \in \mathbb{R}^{n}: g_{t}(x) \leq 0, \forall t \in T\right\}
\end{array}\right.
$$

where

$$
\bar{x} \in \Omega, f_{1}(x):=\max _{1 \leq k \leq p}\left(F_{k}^{L}(x)-F_{k}^{L}(\bar{x})\right) \text { and } f_{2}(x):=\max _{1 \leq k \leq p}\left(F_{k}^{U}(x)-F_{k}^{U}(\bar{x})\right) .
$$

This error has impacted Jennane et al. [6] Lemma 3.4 and Theorem 4.5 which are accordingly false too.

Remark 3.4. In Lemma $3.4[6]$, the inclusion

$$
\{\text { weak minima of Problem (4) }\} \subseteq\{\text { weak efficient solutions of Problem (2) }\}
$$

is not logical and, therefore, lacks validity.

- The structure of Problem (4) requires that $\bar{x}$ be already a weak efficient solution of Problem (2).

- Since Problem (4) depends closely on weak efficient solutions of Problem (2), equality between the set of weak efficient solutions of Problem (2) and that of weak minima of Problem (4) is not logical. 
Remark 3.5. In itself, the Abadie constraint qualification does not necessarily ensure the second part of Remark 4.3 from $[6]$; i.e.

$$
N_{\Omega}(\bar{x})=\operatorname{cl} \text { cone }\left(\bigcup_{t \in T(\bar{x})} \operatorname{co}\left(\partial^{\mathrm{us}} g_{t}(\bar{x})\right)\right)
$$

where

$$
T(\bar{x}):=\left\{t \in T: g_{t}(\bar{x})=0\right\} .
$$

Kabgani and Soleimani-damaneh [8] proved the last equality under the closedness of the set cone $\left(\bigcup_{t \in T(\bar{x})} \partial^{\mathrm{us}} g_{t}(\bar{x})\right)$. For more details, see Theorem 3.3 of [8]. See also Summary of Section 3 from [8].

The following example shows that the set cone $\left(\bigcup_{t \in T(\bar{x})} \partial^{\text {us }} g_{t}(\bar{x})\right)$ is not necessarily closed even if the Abadie constraint qualification is satisfied.

Example 3.6. Let $\bar{x}=(0,0)$ and $T:=[-1,1[$. Let

$$
g_{t}\left(x_{1}, x_{2}\right):=t x_{1}-x_{2}, \forall t \in T
$$

and

$$
\Omega=\left\{\left(x_{1}, x_{2}\right) \in \mathbb{R}^{2}: g_{t}\left(x_{1}, x_{2}\right) \leq 0, \forall t \in T\right\} .
$$

- On the one hand, since

$$
x=\left(x_{1}, x_{2}\right) \in \Omega \Longleftrightarrow g_{t}\left(x_{1}, x_{2}\right) \leq 0, \forall t \in T
$$

we get

$$
x \in \Omega \Longleftrightarrow t x_{1}-x_{2} \leq 0, \forall t \in T .
$$

Then,

$$
\Omega=\left\{\left(x_{1}, x_{2}\right) \in \mathbb{R}^{2}: x_{2}>\left|x_{1}\right|\right\} \cup\left\{\left(x_{1}, x_{2}\right) \in \mathbb{R}^{2}: x_{2}=-x_{1}, x_{2} \geq 0\right\} .
$$

Consequently,

$$
T_{\Omega}(\bar{x})=\left\{\left(x_{1}, x_{2}\right) \in \mathbb{R}^{2}: x_{2} \geq\left|x_{1}\right|\right\} .
$$

Then,

$$
N_{\Omega}(\bar{x})=\left\{\left(x_{1}, x_{2}\right) \in \mathbb{R}^{2}: x_{2} \leq\left|x_{1}\right|\right\} .
$$

- On the other hand, since $T(\bar{x})=T$ and since

$$
\partial^{\mathrm{us}} g_{t}(\bar{x})=\{(t,-1)\}, \forall t \in T
$$

we obtain

$$
\bigcup_{t \in T(\bar{x})} \partial^{\mathrm{us}} g_{t}(\bar{x})=T \times\{-1\}
$$

Consequently,

$$
\left(\bigcup_{t \in T(\bar{x})} \partial^{\mathrm{us}} g_{t}(\bar{x})\right)^{\circ}=\Omega
$$

and the Abadie constraint qualification holds at $\bar{x}$. 
- Since

$$
\text { cone }\left(\bigcup_{t \in T(\bar{x})} \operatorname{co}\left(\partial^{\mathrm{us}} g_{t}(\bar{x})\right)\right)=\left\{\left(x_{1}, x_{2}\right) \in \mathbb{R}^{2}: x_{2}<-\left|x_{1}\right|\right\} \cup\left\{\left(x_{1}, x_{2}\right) \in \mathbb{R}^{2}: x_{2}=x_{1}, x_{2} \leq 0\right\}
$$

we deduce that cone $\left(\bigcup_{t \in T(\bar{x})} \operatorname{co}\left(\partial^{\mathrm{us}} g_{t}(\bar{x})\right)\right)$ is not closed.

Remark 3.7. The example illustrating Theorem 4.5 of [6] is not correct. Since $\bar{x}=(0,0)$ is not a feasible solution of Problem (11) of [6] (see Example 3.2), neither $\bar{x}$ is a weak efficient solution of (11) nor $\Omega$ is locally star-shaped at $\bar{x}$.

\section{Optimality conditions}

Let $x, \bar{x} \in \Omega$ and let $t \in T$. Consider the functions

$$
g_{t}^{L}(x):=G_{t}^{L}(x)-A_{t}^{L}, g_{t}^{U}(x):=G_{t}^{U}(x)-A_{t}^{U} \text { and } g_{t}(x):=\max \left(g_{t}^{L}(x), g_{t}^{U}(x)\right) .
$$

We shall need the following assumptions.

\section{- Assumption 1}

The functions $f_{1}$ and $f_{2}$ defined in (3.1) have USRCs at $\bar{x}$, respectively as

$$
\partial^{\mathrm{us}} f_{1}(\bar{x}) \subseteq \operatorname{co}\left(\bigcup_{k \in I^{L}(\bar{x})} \operatorname{co}\left(\partial^{\mathrm{us}} F_{k}^{L}(\bar{x})\right)\right) \text { and } \partial^{\mathrm{us}} f_{2}(\bar{x}) \subseteq \operatorname{co}\left(\bigcup_{k \in I^{U}(\bar{x})} \operatorname{co}\left(\partial^{\mathrm{us}} F_{k}^{U}(\bar{x})\right)\right),
$$

where

$$
I^{L}(\bar{x})=\left\{k \in I: F_{k}^{L}(\bar{x})=f_{1}(\bar{x})\right\} \text { and } I^{U}(\bar{x})=\left\{k \in I: F_{k}^{U}(\bar{x})=f_{2}(\bar{x})\right\} .
$$

\section{- Assumption 2}

For all $t \in T$, the function $g_{t}$ defined in (4.1) has an USRC at $\bar{x}$ such that

$$
\partial^{\mathrm{us}} g_{t}(\bar{x}) \subseteq \operatorname{co}\left(\partial^{\mathrm{us}} G_{t}^{L}(\bar{x}), \partial^{\mathrm{us}} G_{t}^{U}(\bar{x})\right) .
$$

\section{- Assumption 3}

$$
T^{L}(x) \cap T^{U}(x)=\emptyset, \forall x \in \widetilde{\Omega},
$$

where

$$
\widetilde{\Omega}:=\left\{x \in \mathbb{R}^{n}: g_{t}^{L}(x) \leq 0, g_{t}^{U}(x) \leq 0, \forall t \in T\right\}
$$

and

$$
T^{L}(x)=\left\{t \in T: g_{t}^{L}(x)=0\right\} \text { and } T^{U}(x)=\left\{t \in T: g_{t}^{U}(x)=0\right\} .
$$

\section{- Assumption 4}

The convex cone generated by

$$
\Gamma(\bar{x}):=\bigcup_{t \in T(\bar{x})} \partial^{\mathrm{us}} g_{t}(\bar{x})
$$

is closed. 
Example 4.1. Let $X=R$,

$$
T:=[0,2], G_{t}^{L}(x):=-2 x, G_{t}^{U}(x):=x^{2}-t x+2+t, A_{t}^{L}(x):=2 \text { and } A_{t}^{U}(x):=2+t .
$$

Since

$$
g_{t}^{L}(x):=-2 x-2 \text { and } g_{t}^{U}(x):=x^{2}-t x
$$

we have

$$
T^{L}(x)=\left\{\begin{array}{ll}
T & \text { if } x=-1 \\
\emptyset & \text { if } x \neq-1
\end{array} \text { and } T^{U}(x)= \begin{cases}T & \text { if } x=0 \text { or } x=t \\
\emptyset & \text { otherwise }\end{cases}\right.
$$

Since

$$
\widetilde{\Omega}=\left\{x \in \mathbb{R}: g_{t}(x) \leq 0, \forall t \in T\right\}=\{0\}
$$

we deduce that

$$
T^{L}(x) \cap T^{U}(x)=\emptyset, \forall x \in \widetilde{\Omega}
$$

which means that Assumption 3 is satisfied.

The following result is a corrected version of Lemma 3.3 of [6].

Lemma 4.2. Assume that Assumption 3 is fulfilled. Then, the feasible set $\Omega$ of Problem (2) is equal to

$$
\widetilde{\Omega}=\left\{x \in \mathbb{R}^{n}: g_{t}(x) \leq 0, \forall t \in T\right\} .
$$

Proof. Let $x \in \mathbb{R}^{n}$ and $t \in T$. Since

$$
G_{t}(x) \leq_{L U} A_{t} \Longleftrightarrow\left\{\begin{array}{l}
G_{t}^{L}(x)-A_{t}^{L} \leq 0, \\
G_{t}^{U}(x)-A_{t}^{U} \leq 0, \\
\left(G_{t}^{L}(x)-A_{t}^{L}, G_{t}^{U}(x)-A_{t}^{U}\right) \neq(0,0),
\end{array}\right.
$$

we have

$$
G_{t}(x) \leq_{L U} A_{t} \Longleftrightarrow\left\{\begin{array}{l}
g_{t}^{L}(x) \leq 0, \\
g_{t}^{U}(x) \leq 0, \\
\left(g_{t}^{L}(x), g_{t}^{U}(x)\right) \neq(0,0) .
\end{array}\right.
$$

Assumption 3 implies

$$
G_{t}(x) \leq_{L U} A_{t} \Longleftrightarrow\left\{\begin{array}{l}
g_{t}^{L}(x) \leq 0 \\
g_{t}^{U}(x) \leq 0 .
\end{array} \Longleftrightarrow g_{t}(x) \leq 0\right.
$$

The following result is a corrected version of Lemma 3.4 of [6].

Lemma 4.3. Suppose that Assumption 3 is fulfilled and that $\bar{x} \in \Omega$ is a weak efficient solution of Problem (2). Then, $\bar{x}$ is a weak efficient solution of Problem (4).

Proof. Let $\bar{x} \in \Omega$ be a weak efficient solution of Problem (2).

- By Lemma 4.2, using Assumption 3, we have

$$
\Omega=\left\{x \in \mathbb{R}^{n}: g_{t}(x) \leq 0, \forall t \in T\right\} .
$$

Consequently, $\bar{x}$ is a weak efficient solution of the interval-valued vector program

$$
(Q):\left\{\begin{aligned}
\text { Min } & \left\{F(x)=\left(F_{1}(x), \ldots, F_{p}(x)\right): x \in \Omega\right\} \\
\Omega=\left\{x \in \mathbb{R}^{n}: g_{t}(x) \leq 0, \forall t \in T\right\} &
\end{aligned}\right.
$$


- By contrary, suppose that $\bar{x}$ is not a weak efficient solution of (4). Then, we can find $x \in \Omega$ such that

$$
f_{1}(x)<f_{1}(\bar{x}) \text { and } f_{2}(x)<f_{2}(\bar{x}) .
$$

Since $f_{1}(\bar{x})=f_{2}(\bar{x})=0$, we get

$$
F_{k}^{L}(x)-F_{k}^{L}(\bar{x})<0 \text { and } F_{k}^{U}(x)-F_{k}^{U}(\bar{x})<0, \forall k \in I .
$$

Then,

$$
F_{k}(x)<{ }_{L U} F_{k}(\bar{x}), \forall k \in I .
$$

Thus,

$$
F(x)<_{L U} F(\bar{x}) .
$$

Consequently, $\bar{x}$ is not a weak efficient solutions of $(Q)$ which contradicts the fact that $\bar{x}$ is a weak efficient solution of Problem (2).

Definition 4.4. Let $\bar{x} \in \Omega$ and $\partial^{\text {us }} g_{t}(\bar{x})$ be an USRC of $g_{t}$ for any $t \in T$. We say that the Abadie Constraint Qualification (ACQ) holds at $\bar{x}$ if

$$
\Gamma(\bar{x})^{\circ} \subseteq T_{\Omega}(\bar{x}) .
$$

The following remark is a corrected version of Remark 4.3 [6].

Remark $4.5([8])$. Assume that $\Omega$ is locally star-shaped at $\bar{x}$ and that ACQ holds at $\bar{x}$. Then

$$
\Gamma(\bar{x})^{\circ}=T_{\Omega}(\bar{x}) .
$$

If, in addition, cone $\left(\bigcup_{t \in T(\bar{x})} \partial^{\mathrm{us}} g_{t}(\bar{x})\right)$ is closed, we have

$$
N_{\Omega}(\bar{x})=\operatorname{cl} \text { cone }(\Gamma(\bar{x}))=\operatorname{cl} \text { cone }\left(\bigcup_{t \in T(\bar{x})} \operatorname{co}\left(\partial^{\mathrm{us}} g_{t}(\bar{x})\right)\right) .
$$

The following result is the corrected version of Theorem 4.5 from [6].

Theorem 4.6. Let $\Omega$ be locally star-shaped at $\bar{x} \in \Omega$, and let $F_{k}^{L}, F_{k}^{U}, G_{t}^{L}$ and $G_{t}^{U}, k \in I, t \in T$, admit respectively USRCs $\partial^{\mathrm{us}} F_{k}^{L}(\bar{x}), \partial{ }^{\mathrm{us}} F_{k}^{U}(\bar{x}), \partial{ }^{\mathrm{us}} G_{t}^{L}(\bar{x})$ and $\partial^{\mathrm{us}} G_{t}^{U}(\bar{x})$ at $\bar{x}$. Suppose that $A C Q$ holds at $\bar{x}$, that Assumptions 1-4 are fulfilled. If $\bar{x}$ is a weak efficient solution of Problem (2), then there exist an index set $T^{\prime} \subseteq T(\bar{x})$ with $\left|T^{\prime}\right| \leq n, \alpha \in \mathbb{R}_{+}^{\left|I^{L}(\bar{x})\right|}, \beta \in \mathbb{R}_{+}^{\left|I^{U}(\bar{x})\right|}, \mu \in \mathbb{R}_{+}^{\left|T^{\prime}\right|}, \gamma_{t}^{L} \in \mathbb{R}_{+}^{\left|T^{\prime}\right|}, \gamma_{t}^{U} \in \mathbb{R}_{+}^{\left|T^{\prime}\right|}$ and $\lambda \in \mathbb{R}_{+}^{2}$ with

$$
\lambda_{1}+\lambda_{2}=\sum_{k \in I^{L}(\bar{x})} \alpha_{k}=\sum_{k \in I^{U}(\bar{x})} \beta_{k}=\sum_{t \in T^{\prime}} \gamma_{t}^{L}=\sum_{t \in T^{\prime}} \gamma_{t}^{U}=1
$$

such that

$$
\begin{aligned}
0 \in \operatorname{cl} & {\left[\lambda_{1} \sum_{k \in I^{L}(\bar{x})} \alpha_{k} \operatorname{co}\left(\partial^{\mathrm{us}} F_{k}^{L}(\bar{x})\right)+\lambda_{2} \sum_{k \in I^{U}(\bar{x})} \beta_{k} \operatorname{co}\left(\partial^{\mathrm{us}} F_{k}^{U}(\bar{x})\right)\right.} \\
& \left.+\sum_{t \in T^{\prime}} \gamma_{t}^{L} \operatorname{co}\left(\partial^{\mathrm{us}} G_{t}^{L}(\bar{x})\right)+\sum_{t \in T^{\prime}} \gamma_{t}^{U} \operatorname{co}\left(\partial^{\mathrm{us}} G_{t}^{U}(\bar{x})\right)\right]
\end{aligned}
$$


Proof. Let $\bar{x} \in \Omega$ be a weakly efficient solution of Problem (2). By Lemma 4.3 and Assumption 3, it is also a weak efficient solution of Problem (4). Using Theorem 4.1 of [8], we can find $\lambda_{1}, \lambda_{2} \geq 0, \lambda_{1}+\lambda_{2}=1$ such that

$$
0 \in \operatorname{cl}\left[\lambda_{1} \operatorname{co}\left(\partial^{\mathrm{us}} f_{1}(\bar{x})\right)+\lambda_{2} \operatorname{co}\left(\partial^{\mathrm{us}} f_{2}(\bar{x})\right)+N_{\Omega}(\bar{x})\right] .
$$

Using Assumption 1, we get

$$
0 \in \mathrm{cl}\left[\lambda_{1} \operatorname{co}\left(\bigcup_{k \in I^{L}(\bar{x})} \operatorname{co}\left(\partial^{\mathrm{us}} F_{k}^{L}(\bar{x})\right)\right)+\lambda_{2} \operatorname{co}\left(\bigcup_{k \in I^{U}(\bar{x})} \operatorname{co}\left(\partial^{\mathrm{us}} F_{k}^{U}(\bar{x})\right)\right)+N_{\Omega}(\bar{x})\right] .
$$

Then, there exist $\alpha \in \mathbb{R}_{+}^{\left|I^{L}(\bar{x})\right|}$ and $\beta \in \mathbb{R}_{+}^{\left|I^{U}(\bar{x})\right|}$ such that

$$
\sum_{k \in I^{L}(\bar{x})} \alpha_{k}=\sum_{k \in I^{U}(\bar{x})} \beta_{k}=1
$$

and

$$
0 \in \operatorname{cl}\left[\lambda_{1} \sum_{k \in I^{L}(\bar{x})} \alpha_{k} \operatorname{co}\left(\partial^{\mathrm{us}} F_{k}^{L}(\bar{x})\right)+\lambda_{2} \sum_{k \in I^{U}(\bar{x})} \beta_{k} \operatorname{co}\left(\partial^{\mathrm{us}} F_{k}^{U}(\bar{x})\right)+N_{\Omega}(\bar{x})\right] .
$$

In the light of Remark 4.5, if we use ACQ at $\bar{x}$ and Assumption 4, we obtain an index set $T^{\prime}$ and $\mu \in \mathbb{R}_{+}^{\left|T^{\prime}\right|}$ such that $\left|T^{\prime}\right| \leq n$ and

$$
0 \in \operatorname{cl}\left[\lambda_{1} \sum_{k \in I^{L}(\bar{x})} \alpha_{k} \operatorname{co}\left(\partial^{\mathrm{us}} F_{k}^{L}(\bar{x})\right)+\lambda_{2} \sum_{k \in I^{U}(\bar{x})} \beta_{k} \operatorname{co}\left(\partial^{\mathrm{us}} F_{k}^{U}(\bar{x})\right)+\sum_{t \in T^{\prime}} \mu_{t} \operatorname{co}\left(\partial^{\mathrm{us}} g_{t}(\bar{x})\right)\right] .
$$

Finaly, using Assumption 2 we get the desired result.

\section{Conclusions}

In the paper [6], the authors investigated a semi-infinite interval-valued vector program. The main result, Theorem 4.5 [6] and the intermediate results ([6], Lems. 3.3 and 3.4) on which the authors based their argument are not adequate. In this work, counterexamples are given to call into question Lemmas 3.3 and 3.4 of [6]. Furthermore, we correct the flaws and give a new and short proof.

Acknowledgements. Our sincere acknowledgements to the anonymous referees for their insightful remarks and suggestions.

\section{REFERENCES}

[1] A.K. Bhurjee and G. Panda, Sufficient optimality conditions and duality theory for interval optimization problem. Ann. Oper. Res. 243 (2016) 335-348.

[2] Y. Chalco-Cano, W.A. Lodwick and A. Rufian-Lizana, Optimality conditions of type KKT for optimization problem with interval-valued objective function via generalized derivative. Fuzzy Optim. Decis. Making 12 (2013) 305-322.

[3] J. Dutta and S. Chandra, Convexifactors, generalized convexity, and optimality conditions. J. Optim. Theory App. 113 (2002) 41-64.

[4] G.M. Ewing, Sufficient conditions for global minima of suitably convex functionals from variational and control theory. SIAM Rev. 19 (1977) 202-220.

[5] A. Jayswal, I. Stancu-Minasian and I. Ahmad, On sufficiency and duality for a class of interval-valued programming problems. Appl. Math. Comput. 218 (2011) 4119-4127.

[6] M. Jennane, E. Kalmoun and L. Lafhim, Optimality conditions for nonsmooth interval-valued and multiobjective semi-infinite programming. RAIRO:OR $\mathbf{5 5}$ (2021) 1-11. 
[7] V. Jeyakumar and D.T. Luc, Nonsmooth calculus, minimality, and monotonicity of convexifactors. J. Optim. Theory App. 101 (1999) 599-621.

[8] A. Kabgani and M. Soleimani-damaneh, Characterization of (weakly/properly/robust) efficient solutions in nonsmooth semiinfinite multiobjective optimization using convexificators. Optimization 67 (2017) 217-235.

[9] S. Kaur, Theoretical studies in mathematical programming. Ph.D. thesis. University of Delhi (1983).

[10] H.-C. Wu, On interval-valued nonlinear programming problems. J. Math. Anal. Appl. 338 (2008) 299-316. 